\title{
Synthesized affine invariant function for 2D shape recognition
}

\author{
Wei-Song Lin*, Chun-Hsiung Fang \\ Department of Electrical Engineering, National Taiwan University, No. 1, Sector 4, Roosevelt Road, Taipei 106, Taiwan, ROC
}

Received 12 September 2005; received in revised form 21 February 2006; accepted 31 March 2006

\begin{abstract}
By defining the weighted wavelet synthesis, the synthesized feature signals of an interesting shape are extracted to derive the innovative synthesized affine invariant function (SAIF). The synthesized feature signals hold the shape information with minimum loss by excluding simply the translation dependent and noise-contaminated bands. The SAIF is shown excellent in the invariance property and representative in describing the original shape for automated recognition. Experimental results demonstrate that automated shape recognition based on the SAIF achieves high correctness and significantly outperforms those using conventional wavelet affine invariant functions.

(C) 2006 Pattern Recognition Society. Published by Elsevier Ltd. All rights reserved.
\end{abstract}

Keywords: Affine invariant function; Shape recognition; Wavelet transform; Synthesized feature signal; Weighted wavelet synthesis

\section{Introduction}

Human vision unveils that the shape feature is a significant perception to distinguish different objects. This inspires researchers to find reliable and efficient shape features for discriminating objects by machine vision. Presently, the shape features presented in literature are mainly region- or contour-based technique [1]. Region-based techniques extract the key information to represent the overall region. They seem effective but arduous and domain-dependent. On the contrary, contour-based techniques simply trace the boundary, and ignore the content inside the region. Representing a shape with its contour is much more efficient than with the overall region. But the contour captured by a camera under arbitrary orientation may have been distorted by certain geometric transformations. This distortion can be most appropriately represented by the perspective transformation $[2,3]$. The perspective transformation is simplified and approximated by the affine transformation if the depth of objects along the line of sight is small compared with the viewing distance. The affine transformation includes typical geometric transformations as rotation, translation, scaling, skewing,

\footnotetext{
* Corresponding author. Tel.: +886233663638; fax: +886223638247

E-mail addresses: weisong@cc.ee.ntu.edu.tw (W.-S. Lin), f1921049@ee.ntu.edu.tw (C.-H. Fang).
}

and shearing. Therefore, before the shape recognition, the affine transformation must be removed from the contour signal so as to obtain reliable result. Thus shape recognition algorithms usually use parameterized contour that is compact representational form of the geometric information and independent of the affine transform [4].

A parameterized contour, which represents the original shape and is independent of the affine transformation, is called an affine invariant descriptor or affine invariant function (AIF). Area moment invariants are invariant to similarity transformation except the computing is usually time-consuming $[5,6]$. Except a few low order moment invariants, the affine weighted curve moments [7] and the affine invariant moment utilizing B-spline [8] are highly sensitive to noise. The affine arc length, which is calculated by the first and second derivatives of the object contour [9], is less robust in a noisy environment. The enclosed area, which is based on the property of all area being changed in the same ratio under an affine mapping, is translation dependent [3]. Recently, wavelet transform has been introduced in deriving the AIF and called the wavelet affine invariant function (WAIF). The WAIF usually derives with the coarse and detailed signals obtained in different wavelet transform levels. A WAIF using only one wavelet dyadic level was presented in $[2,10]$. This WAIF, however, is not invariant to translation unless the contour has its centroid as the origin. 
Deriving a WAIF with two or more wavelet dyadic levels is possible $[1,11,12]$. In these situations, the wavelet signals are usually selected to be translation independent. That using three, four, or six wavelet dyadic levels is originated from one conic equation, four collinear points and two conic equations, respectively [13]. Generally speaking, the WAIF is suffered from a difficulty of losing important shape information in the neglected wavelet dyadic levels. Consequently, shape recognition based on the WAIF may be inaccurate and unreliable.

This paper presents the synthesized affine invariant function (SAIF) derived with the synthesized feature signals (SFS) of the shape. A SFS is defined as a weighted wavelet synthesis of the original contour signal. A set of mutually orthogonal SFSs excluding simply the translation dependent and noise-contaminated bands are extracted from the contour signal to derive the SAIF. The SAIF is shown a representational and information-rich form of the original shape for automated recognition.

The remainder of this paper is organized as follows: Section 2 introduces the weighted wavelet synthesis and the SFS of a shape. Section 3 derives two SAIFs with the SFSs for automated shape recognition. Section 4 evaluates experimentally the performance of the SAIF and compares the results with those obtained by the WAIF $[1,12]$ and the summation invariant (SumAIF) [14]. Finally, Section 5 is the conclusion.

\section{Weighted wavelet synthesis and synthesized feature signal}

\subsection{Weighted wavelet synthesis}

In the multiresolution formulation of dyadic wavelet analysis, i.e. wavelet transform, a signal or function $f(\tau) \in \mathbf{V}_{J}$ decomposed to a certain wavelet scale level $i$ can be expressed as [15]

$$
\begin{aligned}
f(\tau)= & \sum_{k=0}^{2^{i}-1} c_{i}(k) 2^{i / 2} \phi\left(2^{i} \tau-k\right) \\
& +\sum_{j=i}^{J} \sum_{k=0}^{2^{j}-1} d_{j}(k) 2^{j / 2} \psi\left(2^{j} \tau-k\right),
\end{aligned}
$$

where $c_{i}$ 's are called the coarse coefficients at level $i, d_{j}^{\prime} s$ are the detailed coefficients at level $j, j$ and $k$ are integer indices, $J$ is an integer denoting the starting or highest scale level, $i$ is an integer denoting the end scale level, $\left\{\phi\left(2^{i} \tau-k\right) \mid \forall k \in N\right\}$ is the set of level- $i$ scaling functions, $\left\{\psi\left(2^{j} \tau-k\right) \mid \forall k \in N\right\}$ is the set of level- $j$ wavelet functions. The set of functions $\left\{\phi\left(2^{i} \tau-k\right), \psi\left(2^{j} \tau-k\right) \mid \forall k \in N\right\}$ forms an orthogonal basis that spans $\mathbf{V}_{J}$. In a practical application of the wavelet analysis, the signal is perhaps itself a sequence of numbers or samples of some function of a continuous variable. In this situation, the scaling coefficients at the starting level are approximated by the corresponding numbers or samples, meaning no wavelet coefficients are necessary at that level. Thus, $c_{J}(k)=f(k), k=0,1,2, \ldots$ and $d_{J}(k)=0$ are appropriate at the starting level $J$. Expansion coefficients at lower levels can then be evaluated by using the following iterative formula [16]:

$c_{j}(k)=\sum_{m} h_{0}(m-2 k) c_{j+1}(m)$,
$d_{j}(k)=\sum_{m} h_{1}(m-2 k) c_{j+1}(m)$,

where $h_{0}(n)$ and $h_{1}(n)$ are called the scaling and wavelet filters, respectively. Conversely, the wavelet synthesis, i.e. wavelet inverse transform, combines the expansion coefficients as follows:

$$
\begin{aligned}
c_{j+1}(k)= & \sum_{m} c_{j}(m) h_{0}(k-2 m) \\
& +\sum_{m} d_{j}(m) h_{1}(k-2 m) .
\end{aligned}
$$

Iterative applications of (4) may ultimately reconstruct the original samples $c_{J}(k)=f(k), k=0,1,2, \ldots$ If the original signal is some function of a continuous variable, the synthesis should be implemented by substituting the expansion coefficients into (1).

The weighted wavelet synthesis is defined in this paper by synthesizing a signal with,

$$
\begin{aligned}
S_{w}(f(\tau))= & w_{c} \sum_{k=0}^{2^{i}-1} c_{i}(k) 2^{i / 2} \phi\left(2^{i} \tau-k\right) \\
& +\sum_{j=i}^{J} w_{j} \sum_{k=0}^{2^{j}-1} d_{j}(k) 2^{j / 2} \psi\left(2^{j} \tau-k\right),
\end{aligned}
$$

where $w_{c}$ is called the coarse weight, $\left\{w_{j} \mid j=i, \ldots, J\right\}$ are called the detailed weights, and $\mathbf{w}=\left\{w_{c}\right.$, all $\left.w_{j}\right\}$ is the set of weights to be designed. Associating each designed weight in $\mathbf{w}$ with the corresponding term in (4) obtains the following iterative formula of the weighted wavelet synthesis:

$$
\begin{aligned}
S_{w}\left(c_{j+1}(k)\right)= & w_{c j} \sum_{m} S_{w}\left(c_{j}(m)\right) h_{0}(k-2 m) \\
& +w_{j} \sum_{m} d_{j}(m) h_{1}(k-2 m),
\end{aligned}
$$

where $w_{c j}=w_{c}$, as $j=i$ otherwise $w_{c j}=1$. Apparently, setting one to each weight in $\mathbf{w}$ will recover the wavelet synthesis. Alternatively, the weights $\mathbf{w}$ can be designed so that the weighted wavelet synthesis obtains signal $S_{w}(f(\tau))$ or $S_{w}\left(c_{J}(k)\right)$ with interesting property such as translation independent, noise free or mutually orthogonal. This characteristic is very useful in deriving the AIF for shape recognition. 


\subsection{Synthesized feature signals of a shape}

In the contour-based technique, a shape is described by a contour signal. Applying the weighted wavelet synthesis to the contour signal and adapting the weights may obtain a derivative contour signal simply consisting of interesting wavelet bands of the original contour signal. A set of such derivative contour signals with mutually orthogonal and translation independent properties and possibly noise free is called the SFS of the shape. Translation independency can be achieved by discarding the coarse weight $w_{c}$ in the weighted wavelet synthesis. Neglecting the weights corresponding to noise-contaminated bands can eliminate noise. In addition, since the wavelet transform with orthogonal basis is used, weight sets consist of non-overlapping bands may produce a set of orthogonal derivative signals. The orthogonal property makes the SFSs of the shape free from redundant information and therefore computationally efficient.

Exclusive weighting is a simple method to design the weight sets to synthesize the SFSs of a shape. The exclusive weighting method is described as follows:

(1) Build the weight set $\mathbf{w}=\left\{w_{c}\right.$, all $\left.w_{j}\right\}$ associated with (5). Then construct the mother weight set by discarding (set to zero) the coarse weight $w_{c}$ for translation independency, discarding any $w_{j}$ corresponding to the noise-contaminated band, and adjust the rest of $w_{j}$ 's to the desirable strength (usually nonzero and one in our design).

(2) Split the mother weight set into constant dimensional, non-null, exclusive weight subsets as many as the desirable number of the SFSs. The union of these exclusive weight subsets must equal to the mother weight set so that no information loss is ensured. The intersection of these exclusive weight subsets is null. This ensures the SFSs obtained by applying these exclusive weight subsets to (5) are mutually orthogonal.

Consequently, except the translation dependent and nosecontaminated bands, all the shape information is kept in the SFSs or, in other words, the SFSs represent the original shape with minimum information loss.

\section{Synthesized affine invariant functions}

\subsection{Relative and absolute affine invariant functions of a contour}

Affine transformation of an object contour can be expressed as the following matrix form of mapping functions:

$$
\begin{aligned}
{\left[\begin{array}{c}
\tilde{x}(\tau) \\
\tilde{y}(\tau)
\end{array}\right] } & =\left[\begin{array}{ll}
a_{11} & a_{12} \\
a_{21} & a_{22}
\end{array}\right]\left[\begin{array}{l}
x(\tau) \\
y(\tau)
\end{array}\right]+\left[\begin{array}{l}
b_{1} \\
b_{2}
\end{array}\right] \\
& =\mathbf{A}\left[\begin{array}{l}
x(\tau) \\
y(\tau)
\end{array}\right]+\mathbf{B},
\end{aligned}
$$

where $(x(\tau), y(\tau))$ represents a point on the contour parameterized by the arc length parameter $\tau,(\tilde{x}(\tau), \tilde{y}(\tau))$ is the corresponding point after the affine transformation, $\mathbf{A}$ is a nonsingular square matrix representing the rotation, scaling, and skewing transformations, and the vector $\mathbf{B}$ represents the translation. The affine transformation matrix $\mathbf{A}$ can also be expressed by several parameters as follows:

$\mathbf{A}=$ scale $\cdot\left[\begin{array}{cc}\cos \theta & -\sin \theta \\ \sin \theta & \cos \theta\end{array}\right]\left[\begin{array}{cc}1 & x_{\text {skew }} \\ y_{\text {skew }} & 1\end{array}\right]$,

where scale is the scale factor, $\theta$ is the rotation angle, and $x_{\text {skew }}$ and $y_{\text {skew }}$ are the skewing parameters in $x$ - and $y$ directions, respectively. The characteristic within the affine transformation is that parallel lines map to parallel lines, i.e. a square can map onto an arbitrary parallelogram.

If $\delta$ is an AIF, and $\tilde{\delta}$ is the corresponding invariant function calculated by the points under the affine transformation, the relation can be denoted as [1]

$\tilde{\delta}=\delta|\mathbf{A}|^{\rho}$,

where $|\cdot|$ denotes the determinant and $\rho$ is called the weight of the invariance. The AIF is called an absolute invariant as $\rho=0$ and a relative invariant as $\rho \neq 0$.

\subsection{Deriving the synthesized affine invariant functions}

Let $\left(S_{\alpha}(x), S_{\alpha}(y)\right)$ and $\left(S_{\beta}(x), S_{\beta}(y)\right)$ be the SFSs of a shape, then the affine transformation mapping function is represented as

$$
\begin{aligned}
{\left[\begin{array}{ll}
S_{\alpha}(\tilde{x}) & S_{\beta}(\tilde{x}) \\
S_{\alpha}(\tilde{y}) & S_{\beta}(\tilde{y})
\end{array}\right]=} & {\left[\begin{array}{ll}
a_{11} & a_{12} \\
a_{21} & a_{22}
\end{array}\right]\left[\begin{array}{ll}
S_{\alpha}(x) & S_{\beta}(x) \\
S_{\alpha}(y) & S_{\beta}(y)
\end{array}\right] } \\
& +\left[\begin{array}{ll}
S_{\alpha}\left(b_{1}\right) & S_{\beta}\left(b_{1}\right) \\
S_{\alpha}\left(b_{2}\right) & S_{\beta}\left(b_{2}\right)
\end{array}\right] .
\end{aligned}
$$

Eq. (10) can be obtained easily by applying the wavelet analysis to (7) and then followed by the weighted wavelet synthesis. From the translation-independent condition, $S_{\alpha}\left(b_{1}\right)=$ $S_{\beta}\left(b_{1}\right)=S_{\alpha}\left(b_{2}\right)=S_{\beta}\left(b_{2}\right)=0,(10)$ is rewritten as

$\left[\begin{array}{ll}S_{\alpha}(\tilde{x}) & S_{\beta}(\tilde{x}) \\ S_{\alpha}(\tilde{y}) & S_{\beta}(\tilde{y})\end{array}\right]=\left[\begin{array}{ll}a_{11} & a_{12} \\ a_{21} & a_{22}\end{array}\right]\left[\begin{array}{ll}S_{\alpha}(x) & S_{\beta}(x) \\ S_{\alpha}(y) & S_{\beta}(y)\end{array}\right]$.

Based on the determinant properties and using (9), a relative $\mathrm{SAIF}$ is defined as

$$
\mu_{\alpha, \beta}(\tau)=S_{\alpha}[x(\tau)] S_{\beta}[y(\tau)]-S_{\alpha}[y(\tau)] S_{\beta}[x(\tau)] .
$$

Normalization of (12) obtains the following absolute SAIF:

$$
\begin{aligned}
\hat{\mu}_{\alpha, \beta}(\tau) & =\frac{\mu_{\alpha, \beta}(\tau)}{\mu_{\alpha, \beta}\left(\tau^{*}\right)} \\
& =\frac{S_{\alpha}[x(\tau)] S_{\beta}[y(\tau)]-S_{\alpha}[y(\tau)] S_{\beta}[x(\tau)]}{S_{\alpha}\left[x\left(\tau^{*}\right)\right] S_{\beta}\left[y\left(\tau^{*}\right)\right]-S_{\alpha}\left[y\left(\tau^{*}\right)\right] S_{\beta}\left[x\left(\tau^{*}\right)\right]},
\end{aligned}
$$

where $\mu_{\alpha, \beta}\left(\tau^{*}\right)$ represents the maximum of $\mu_{\alpha, \beta}(\tau)$, and $\tau^{*}$ denotes the instant of occurrence. 
Let $\left(S_{\alpha}(x), S_{\alpha}(y)\right),\left(S_{\beta}(x), S_{\beta}(y)\right)$ and $\left(S_{\gamma}(x), S_{\gamma}(y)\right)$ be the SFSs of a shape, then another SAIF can be derived by using the conic equation. The conic is a curve defined directly in terms of a projective invariant property. Given a point of the contour signal, the conic can be expressed as the quadratic form, which is symmetric, defined by

$\left[\begin{array}{l}x(\tau) \\ y(\tau)\end{array}\right]^{\mathrm{T}}\left[\begin{array}{ll}v_{11} & v_{12} \\ v_{12} & v_{22}\end{array}\right]\left[\begin{array}{l}x(\tau) \\ y(\tau)\end{array}\right]=1$

The AIF is defined as $v_{11} v_{22}-v_{12}^{2}$ [13]. Thus, a relative SAIF with the three SFSs is defined as

$$
\begin{aligned}
\mu_{\alpha, \beta, \gamma}(\tau)= & v_{11}(\tau) v_{22}(\tau)-v_{12}^{2}(\tau) \\
= & \left|\begin{array}{lll}
1 & 2 S_{\alpha}[x(\tau)] S_{\alpha}[y(\tau)] & S_{\alpha}^{2}[y(\tau)] \\
1 & 2 S_{\beta}[x(\tau)] S_{\beta}[y(\tau)] & S_{\beta}^{2}[y(\tau)] \\
1 & 2 S_{\gamma}[x(\tau)] S_{\gamma}[y(\tau)] & S_{\gamma}^{2}[y(\tau)]
\end{array}\right| \\
& \times\left|\begin{array}{lll}
S_{\alpha}^{2}[x(\tau)] & 2 S_{\alpha}[x(\tau)] S_{\alpha}[y(\tau)] & 1 \\
S_{\beta}^{2}[x(\tau)] & 2 S_{\beta}[x(\tau)] S_{\beta}[y(\tau)] & 1 \\
S_{\gamma}^{2}[x(\tau)] & 2 S_{\gamma}[x(\tau)] S_{\gamma}[y(\tau)] & 1
\end{array}\right| \\
& -\left|\begin{array}{lll}
S_{\alpha}^{2}[x(\tau)] & 1 & S_{\alpha}^{2}[y(\tau)] \\
S_{\beta}^{2}[x(\tau)] & 1 & S_{\beta}^{2}[y(\tau)] \\
S_{\gamma}^{2}[x(\tau)] & 1 & S_{\gamma}^{2}[y(\tau)]
\end{array}\right|
\end{aligned}
$$

Normalization of (15) obtains the following absolute SAIF:

$$
\begin{aligned}
\hat{\mu}_{\alpha, \beta, \gamma}(\tau) & =\frac{\mu_{\alpha, \beta, \gamma}(\tau)}{\mu_{\alpha, \beta, \gamma}\left(\tau^{*}\right)} \\
& =\frac{v_{11}(\tau) v_{22}(\tau)-v_{12}^{2}(\tau)}{v_{11}\left(\tau^{*}\right) v_{22}\left(\tau^{*}\right)-v_{12}^{2}\left(\tau^{*}\right)},
\end{aligned}
$$

where $\mu_{\alpha, \beta, \gamma}\left(\tau^{*}\right)$ represents the maximum of $\mu_{\alpha, \beta, \gamma}(\tau)$, and $\tau^{*}$ denotes the instant of occurrence. Theoretically, it is possible to derive other SAIF using more than three SFSs. But the computational complexity is a concern. Since the SFSs represent the original shape with minimum information loss. Consequently the SAIF based on the SFSs is a reliable representative of the original shape for automated recognition. On the other hand, conventional WAIFs simply choose several wavelet bands to derive the invariant function. They therefore suffer from a difficulty of loosing important shape information in the neglected bands.

\section{Experimental results}

In the following experiments, the performance of the SAIF is tested by applying to 10 electronic devices and the results are compared with those obtained by conventional WAIF and the SumAIF. The SAIF using two and three SFSs are denoted by $\mathrm{SAIF}_{2}$ and $\mathrm{SAIF}_{3}$, respectively. Conventional WAIF $[1,12]$ using two, three and six dyadic wavelet levels are denoted by $\mathrm{WAIF}_{2}, \mathrm{WAIF}_{3}$ and $\mathrm{WAIF}_{6}$, respectively. The summation invariant [14] is denoted by SumAIF.
Fig. 1(a) shows a photo of the test devices. Among them the M1, M2, M3 and M4 are rectangular integrated circuit devices with different numbers of pins. The image of each device is captured by a digital camera and preprocessed for noise removal and image enhancement before the contour extraction. As illustrated in Fig. 1(b), each contour signal is represented by a 256-point coordinate vector and saved in the contour database. The performances of the SAIF, WAIF and SumAIF are evaluated and compared by examining the functional trajectories, root mean square errors (RMS), local invariance measure (LIM), representative index (RI) and correctness of recognition. However, it should be noticed that the correctness of recognition is dependent of not only the AIF but also the discriminant function.

Let $C$ be a set of contour signals taken from the same shape but undergoing different affine transformations. Then for $i$, ref $\in C$ the RMS and LIM are defined as follows:

$$
\begin{aligned}
\mathrm{RMS} & =\sqrt{\frac{\sum_{\tau=1}^{n}\left(\mathrm{AIF}_{i}(\tau)-\mathrm{AIF}_{r e f}(\tau)\right)^{2}}{n},} \\
\mathrm{LIM} & =\left\|\operatorname{AIF}_{i}-\operatorname{AIF}_{r e f}\right\|_{\infty} \\
& =\max _{\tau}\left|\operatorname{AIF}_{i}(\tau)-\operatorname{AIF}_{r e f}(\tau)\right|,
\end{aligned}
$$

where $n$ is the length of the contour signal, $\tau$ is the arc length parameter and 'ref' denotes the reference signal. The RMS represents general difference between the reference and another contour signal of the same shape. On the other hand, the LIM indicates the worst case difference. The RMS and LIM together can show the stability of an AIF in representing a shape undergoing variant affine transformations. Smaller RMS and LIM imply better invariance property.

Let $S$ be a set of contour signals extracted from different shapes. Then for $i$, target $\in S$, the RI is defined as follows:

$$
\begin{aligned}
\mathrm{RI} & =\frac{\left\|\operatorname{AIF}_{i}(\tau)-\operatorname{AIF}_{\text {target }}(\tau)\right\|_{2}}{\left\|\operatorname{AIF}_{\text {target }}(\tau)\right\|_{2}} \\
& =\sqrt{\frac{\sum_{\tau}\left(\operatorname{AIF}_{i}(\tau)-\operatorname{AIF}_{\text {target }}(\tau)\right)^{2}}{\sum_{\tau} \operatorname{AIF}_{\text {target }}^{2}(\tau)}},
\end{aligned}
$$

where "target" denotes the target shape to be recognized. The denominator can be explained as the energy of the target shape. Accordingly, the numerator represents the energy difference between the target and another shape. The RI indicates the ability of an AIF in discriminating different shapes. Greater RI makes the target shape to be recognized easily from a set of similar shapes.

\subsection{Experiment 1: invariance property of the SAIF}

The contour signal of the device M5 in Fig. 1 is chosen arbitrarily as the reference. Five associative contour signals obtained by applying translation, scaling, rotation, skewing or general affine transform to the M5 contour signal are shown as the first column in Fig. 2. Applying the $\mathrm{SAIF}_{2}$, 


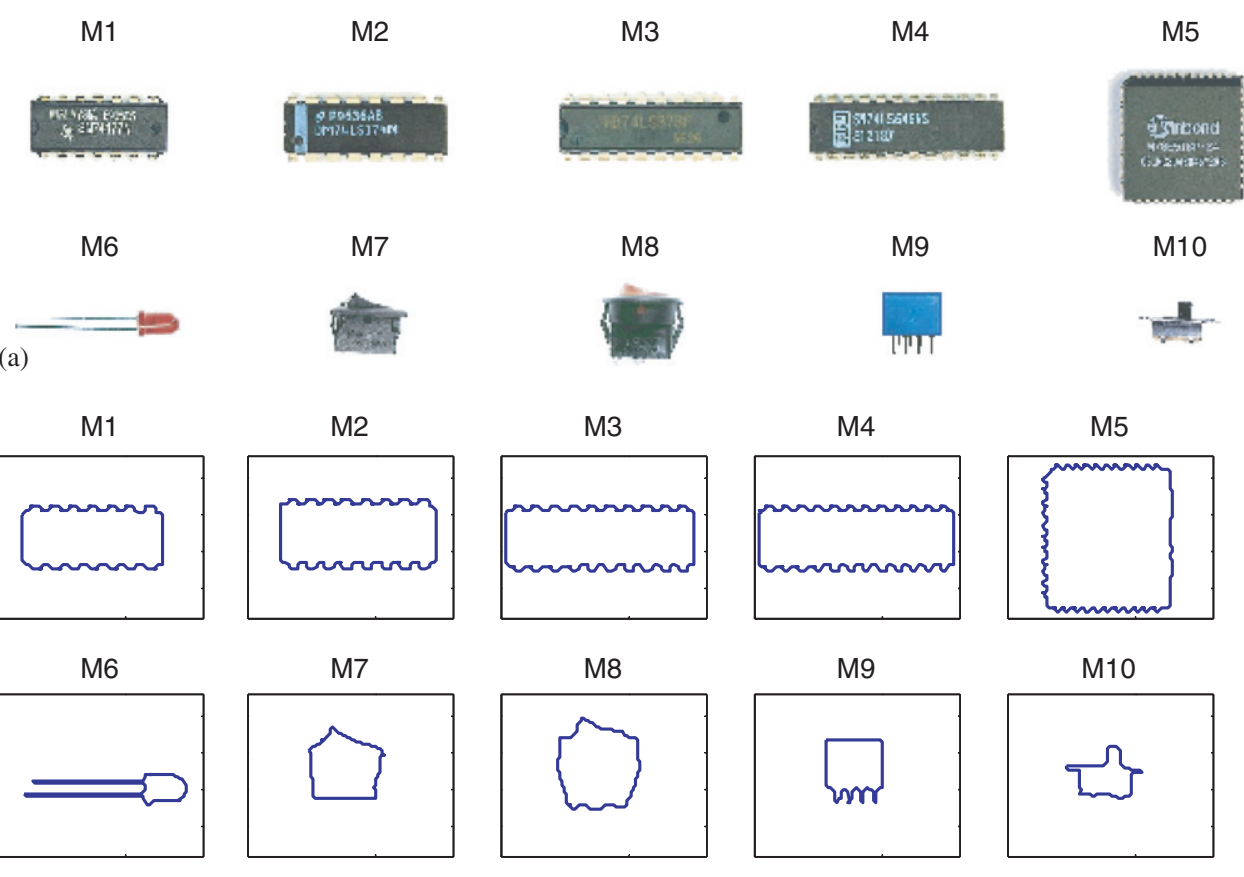

(b)

Fig. 1. (a) A photo of the test devices. (b) Contour signals of the test devices.

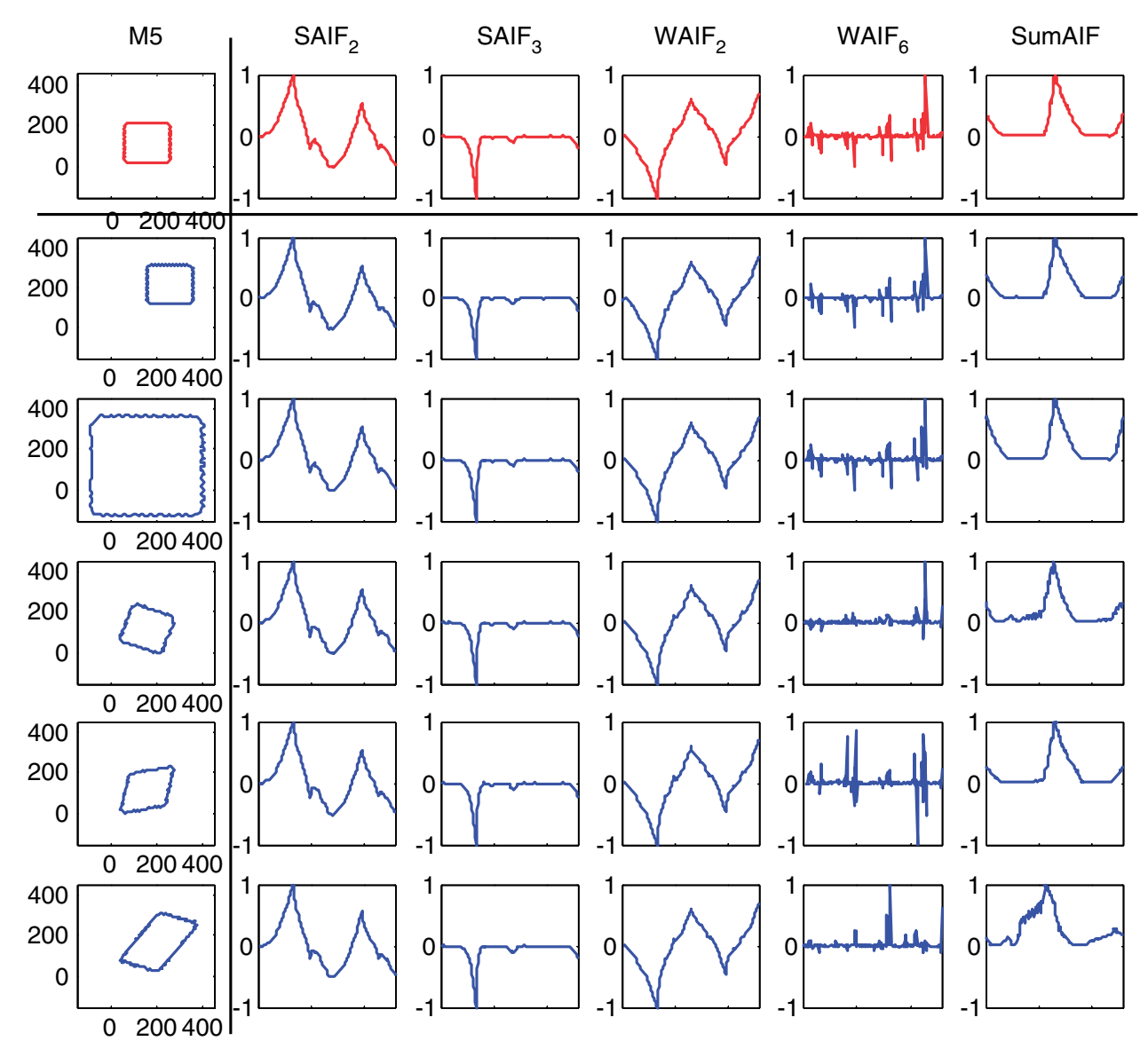

Fig. 2. The AIF trajectories of the device M5 and its associative contour signals. 
Table 1

The RMS values of the associative contour signals of the device M5

\begin{tabular}{llllll}
\hline Affine & AIF & & & \\
\cline { 2 - 6 } & $\mathrm{SAIF}_{2}$ & $\mathrm{SAIF}_{3}$ & WAIF $_{2}$ & WAIF $_{6}$ & SumAIF \\
\hline Translation & 0.0000 & 0.0000 & 0.0000 & 0.0000 & 0.0000 \\
Scaling & 0.0009 & 0.0008 & 0.0000 & 0.0271 & 0.0918 \\
Rotation & 0.0053 & 0.0033 & 0.0027 & 0.0984 & 0.1282 \\
Skewing & 0.0093 & 0.0070 & 0.0046 & 0.1401 & 0.0422 \\
General aff. & 0.0122 & 0.0081 & 0.0009 & 0.1304 & 0.3366 \\
\hline
\end{tabular}

Table 2

The LIM values of the associative contour signals of the device M5

\begin{tabular}{|c|c|c|c|c|c|}
\hline \multirow[t]{2}{*}{ Affine } & \multicolumn{5}{|l|}{ AIF } \\
\hline & $\mathrm{SAIF}_{2}$ & $\mathrm{SAIF}_{3}$ & $\mathrm{WAIF}_{2}$ & $\mathrm{WAIF}_{6}$ & SumAIF \\
\hline Translation & 0.0000 & 0.0000 & 0.0000 & 0.0000 & 0.0000 \\
\hline Scaling & 0.0033 & 0.0061 & 0.0001 & 0.2694 & 0.3561 \\
\hline Rotation & 0.0193 & 0.0203 & 0.0069 & 0.6550 & 0.3910 \\
\hline Skewing & 0.0460 & 0.0451 & 0.0118 & 0.9826 & 0.1874 \\
\hline General aff. & 0.0460 & 0.0654 & 0.0021 & 1.0008 & 0.9212 \\
\hline
\end{tabular}

$\mathrm{SAIF}_{3}, \mathrm{WAIF}_{2}, \mathrm{WAIF}_{6}$, and SumAIF, respectively, to each of these contour signals, the resulting trajectories are presented in Fig. 2 from the 2nd to the 6th columns. In one column, perfect invariance property should present identical trajectories. The similarity between the reference and other trajectories is examined with the RMS and LMI values listed in Tables 1 and 2, respectively. Since the smaller RMS and LIM mean the better invariance property. The $\mathrm{SAIF}_{2}, \mathrm{SAIF}_{3}$ and $\mathrm{WAIF}_{2}$, which present all RMS and LIM values smaller than 0.05 and 0.07 , respectively are satisfactory in experience and significantly better than the $\mathrm{WAIF}_{6}$ and SumAIF. Therefore, the $\mathrm{SAIF}_{2}, \mathrm{SAIF}_{3}$ and $\mathrm{WAIF}_{2}$ perform well in these aspects.

\subsection{Experiment 2: representative property of the SAIF}

To obtain correct recognition, a qualified AIF should produce significantly representative trajectory for each shape. The RI is used to examine the significance of representative of the AIF. Fig. 1 shows the devices M1, M2, M3, and M4 have very similar shapes. Their corresponding AIF trajectories are calculated and presented in Fig. 3. For an AIF, it is found the trajectories of the M1, M2, M3, and M4 are mostly too close to be discriminated by inspection. Let the target of recognition be the M1 then the RI values of the M2, M3, and M4 are calculated for each AIF. Table 3 lists the results. Since the greater RI value means the better representative. Table 3 shows the WAIF $_{6}$ outperforms the others and the $\mathrm{WAIF}_{2}$ is obviously the worst and less reliable. $\mathrm{The} \mathrm{SAIF}_{2}, \mathrm{SAIF}_{3}$ and SumAIF perform in between and the least RI value is 0.1859 . That means at least the target is different from the test by $18.59 \%$ in energy. Therefore, they are satisfactory for discriminating different shapes correctly.

Since an AIF for shape recognition should be qualified both in the invariance and representative properties. An overall evaluation drawn from the results of Experiments 1 and 2 is listed in Table 4. It is found that the WAIF may be good in either invariance or representative property but not both. The worst case condition makes the WAIF not generally

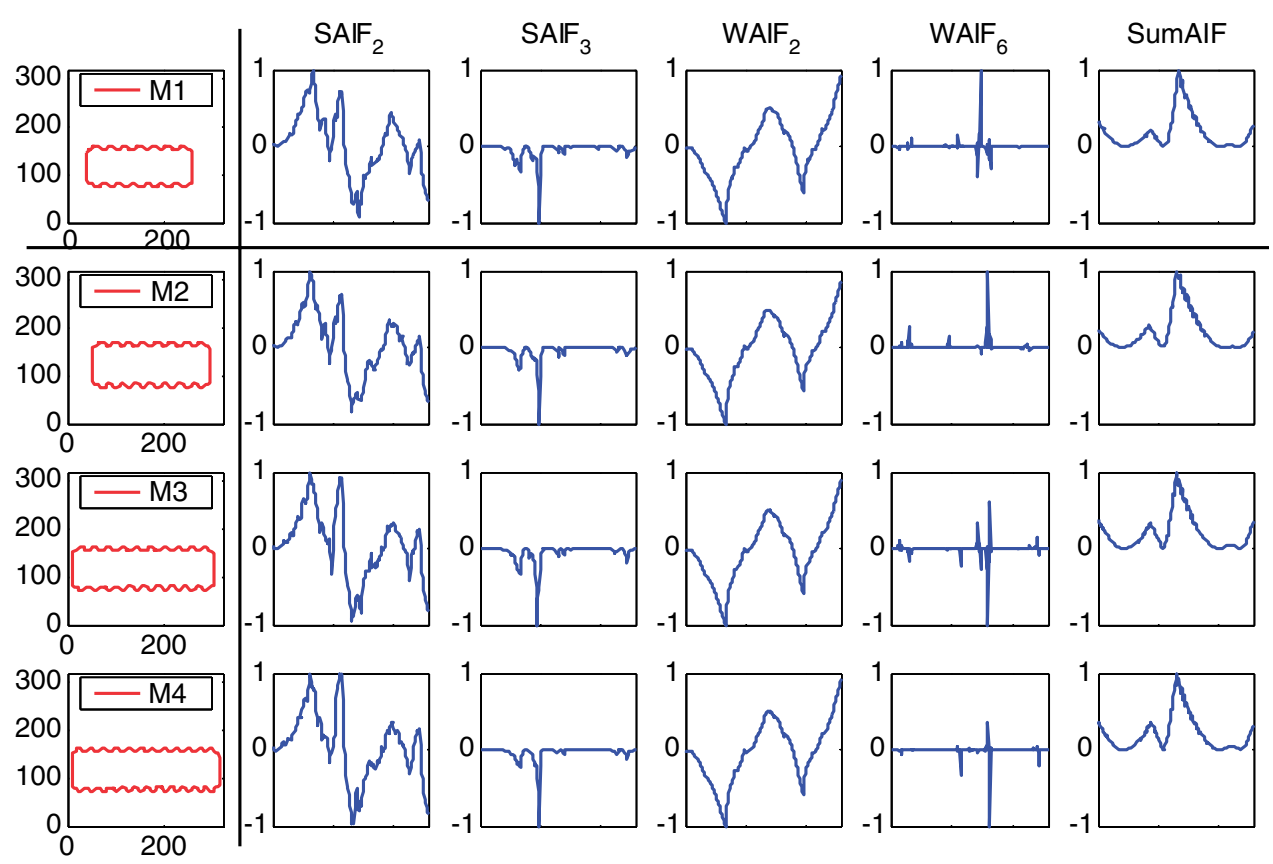

Fig. 3. The AIF trajectories of the devices M1, M2, M3, and M4. 
Table 3

The representative indices of the devices M2, M3, and M4 with respect to the M1

\begin{tabular}{llllll}
\hline Model & \multicolumn{1}{l}{ AIF } & & & \\
\cline { 2 - 6 } & SAIF $_{2}$ & SAIF $_{3}$ & WAIF $_{2}$ & WAIF $_{6}$ & SumAIF \\
\hline M2 & 0.1859 & 0.1876 & 0.0673 & 1.4692 & 0.2173 \\
M3 & 0.2355 & 0.7089 & 0.0293 & 1.6348 & 0.2382 \\
M4 & 0.2637 & 0.2795 & 0.0169 & 1.3645 & 0.2471 \\
\hline
\end{tabular}

appropriate for automated shape recognition. Actually, the performance of the WAIF depends closely on the selection of the frequency bands and may be different from shape to shape. Experiments 1 and 2 conclude that the $\mathrm{SAIF}_{2}$ and $\mathrm{SAIF}_{3}$ are both qualified for the invariance and representative. But the $\mathrm{SAIF}_{2}$ is more computationally efficient. In general, the SAIF outperforms the $\mathrm{WAIF}_{2}, \mathrm{WAIF}_{6}$ and SumAIF in the overall performance.

\subsection{Experiment 3: correctness of shape recognition}

In this experiment, the $\mathrm{SAIF}_{2}, \mathrm{SAIF}_{3}, \mathrm{WAIF}_{2}, \mathrm{WAIF}_{3}$, WAIF $_{6}$, and SumAIF are compared by applying to recognize target shapes out of 2400 contour signals. The thousands of contour signals are obtained by applying 240 different affine transformations to the 10 shapes in Fig. 1. The 240 affine transformations are described by (7) and (8) with scale $\in\{\sqrt{2}, 1,1 / \sqrt{2}\}, \theta \in\left\{0^{\circ}, 36^{\circ}, 72^{\circ}, \ldots, 324^{\circ}\right\}$, skew $\in\{0,0.3\}$, and $\mathbf{B}=[0,200]^{\mathrm{T}}$. Each transformed shape is resampled to obtain a 256-point contour signal. The discrimination function of the shape recognition is chosen as the following similarity measure.

Similarity $\left(\operatorname{AIF}_{M}, \operatorname{AIF}_{T}\right)=\frac{\sum_{k=1}^{N-1} \operatorname{AIF}_{M}(k) \operatorname{AIF}_{T}(k)}{\left\|\operatorname{AIF}_{M}(k)\right\|_{2}\left\|\operatorname{AIF}_{T}(k)\right\|_{2}}$,

Eq. (20) is actually a correlation function in which subscripts " $M$ " and " $T$ " indicate the model and the test contour signals, respectively. In the shape recognition algorithm, a test contour signal is associated with each of the 2400 contour signals to calculate each similarity measure. The model contour signal (one of the 2400 signals) with the greatest similarity measure is regarded as a match and the corresponding shape is the result of recognition. Each of the 2400 contour signals is selected in turn as the test contour signal. Then the same procedure is done for the 2400 contour signals contaminated by Gaussian white noise. The 50 and $30 \mathrm{~dB}$ signal to noise ratio (SNR) cases are calculated and presented. Table 5 lists the statistical results of correctness of shape recognition. Apparently, the $\mathrm{SAIF}_{2}$ is the best. The $\mathrm{SAIF}_{3}$ is the runner up. More multiplications and truncations in calculating the $\mathrm{SAIF}_{3}$ may be the cause of worse performance. Generally speaking the high invariance and representative properties of the $\mathrm{SAIF}_{2}$ and $\mathrm{SAIF}_{3}$ make the automated recognition so accurate. On the other hand, the WAIF simply selects some frequency bands to derive the invariant function. Due
Table 4

Overall performances of the affine invariant functions

\begin{tabular}{llll}
\hline & Invariance & Representative & Overall performance \\
\hline SAIF $_{2}$ & Good & Good & Good \\
SAIF $_{3}$ & Good & Good & Good \\
WAIF $_{2}$ & Good & Bad & Bad \\
WAIF $_{6}$ & Bad & Excellent & Bad \\
SumAIF & Bad & Good & Bad \\
\hline
\end{tabular}

Table 5

Correctness of recognition out of 2400 contour signals

\begin{tabular}{llll}
\hline Correctness $(\%)$ & Noiseless & $\begin{array}{l}\text { Small white noise } \\
(50 \mathrm{~dB} \text { SNR })\end{array}$ & $\begin{array}{l}\text { Large white noise } \\
(30 \mathrm{~dB} \text { SNR })\end{array}$ \\
\hline SAIF $_{2}$ & 95.58 & 95.50 & 93.67 \\
$\mathrm{SAIF}_{3}$ & 94.83 & 94.79 & 82.67 \\
$\mathrm{WAIF}_{2}$ & 72.67 & 72.83 & 59.08 \\
WAIF $_{3}$ & 34.50 & 33.79 & 30.42 \\
WAIF $_{6}$ & 54.67 & 47.13 & 18.79 \\
SumAIF & 18.58 & 18.50 & 18.08 \\
\hline
\end{tabular}

to some distinct shape information may not be contained in the selected bands. Either the invariance or representative property may be deteriorated. As a result, less correctness of recognition is obtained. Although the $\mathrm{WAIF}_{6}$, which includes six dyadic wavelet levels, should be more information rich than the $\mathrm{WAIF}_{2}$ and $\mathrm{WAIF}_{3}$, the weak signals from high dyadic wavelet levels make the $\mathrm{WAIF}_{6}$ unstable. As a consequence, the WAIF $_{6}$ performs no better than its brothers. As for SumAIF, the results in Table 5 show it seems not a good choice for automated shape recognition.

\section{Conclusion}

Based on the well-established technique of wavelet transform, the weighted wavelet synthesis was defined for extracting the SFSs out of a shape. Using the SFSs, the innovative SAIF was derived to represent the original shape for automated shape recognition. The SFSs of a shape was shown to hold the shape information with minimum loss by excluding simply the undesirable bands. The SAIF was shown to have excellent invariance property under variant affine transformations. The SAIF was also shown to have nice representative property for discriminating different shapes. Three experiments have been conducted to verify the SAIF in the invariance property, representative property and reliability in the automated shape recognition, respectively. The SAIF was confirmed to be highly reliable and the automated shape recognition based on it achieved high correctness of recognition. Experimental results also compared the SAIF with the conventional wavelet affine invariant function and the summation invariant. The conclusion was that the power of the SAIF was excellent and second to none of the conventional WAIF and the SumAIF. 


\section{Acknowledgements}

The financial support for this research from the National Science Council of Taiwan, ROC under grants NSC932218-E002-106 and NSC94-2218-E002-049 is gratefully acknowledged.

\section{References}

[1] M.I. Khalil, M.M. Bayoumi, A dyadic wavelet affine invariant function for 2D shape recognition, IEEE Trans. Pattern Anal. Mach. Intell. 23 (10) (2001) 1152-1164.

[2] Q.M. Tieng, W.W. Boles, Wavelet-based affine invariant representation: a tool for recognizing planar objects in 3D space, IEEE Trans. Pattern Anal. Mach. Intell. 19 (8) (1997) 846-857.

[3] K. Arbter, W.E. Snyder, H. Burkhardt, G. Hirzinger, Application of affine-invariant Fourier descriptors to recognition of 3-D objects, IEEE Trans. Pattern Anal. Mach. Intell. 12 (7) (1990) 640-647.

[4] Q.M. Tieng, W.W. Boles, Object recognition using an affine invariant wavelet representation, in: Proceedings of the Second Australia and New Zeland Conference on Intelligent Information Systems, Brisbane, Australia, 1994, pp. 307-311.

[5] M.K. Hu, Visual pattern recognition by moment invariants, IRE Trans. Inform. Theory 12 (1962) 179-187.

[6] J. Flusser, T. Suk, Pattern recognition by affine moment invariants, Pattern Recognition 26 (1993) 167-174.
[7] D. Zhao, J. Chen, Affine curve moment invariants for shape recognition, Pattern recognition 30 (6) (1997) 895-901.

[8] Z. Huang, F.S. Cohen, Affine-invariant B-spline moments for curve matching, IEEE Trans. Image Process. 5 (10) (1996) 1473-1480.

[9] D. Cyganski, R.F. Vaz, A linear signal decomposition approach to affine contour identification, SPIE: Intell. Robots Comput. Vision X 1607 (1991) 98-109.

[10] Q.M. Tieng, W.W. Boles, An application of wavelet-based affine invariant representation, Pattern Recognition Anal. 16 (12) (1995) 1287-1296.

[11] R. Alferez, Y.F. Wang, Geometric and illumination invariants for object recognition, IEEE Trans. Pattern Anal. Mach. Intell. 21 (6) (1999) 505-536.

[12] M.I. Khalil, M.M. Bayoumi, Affine invariants for object recognition using the wavelet transform, Pattern Recognition Lett. 23 (13) (2002) $57-72$.

[13] I. Weiss, Geometric invariants and object recognition, Int. J. Comput. Vision 10 (3) (1993) 207-231.

[14] W.Y. Lin, N. Boston, Y.H. Hu, Summation invariant and its applications to shape recognition, in: Proceedings of the International Conference on Acoustics, Speech, and Signal Processing (ICASSP), vol. 5, 2005, pp. 18-23.

[15] C.S. Burrus, A.R. Gopinath, H. Guo, Introduction to Wavelets and Wavelet Transforms, Prentice-Hall, Englewood Cliffs, NJ, 1998 pp. $1-164$.

[16] S.G. Mallat, A theory for multiresolution signal decomposition: the wavelet representation, IEEE Trans. Pattern Anal. Mach. Intell. 11 (7) (1989) 674-693.

About the Author-WEI-SONG LIN is a professor with the Department of Electrical Engineering, National Taiwan University, Taiwan, ROC. He led the sensor calibration team of Ocean Color Imager aboard FORMOSA-1 (previously called ROCSAT-1) satellite from 1997 to 2001 . His research interests are computational intelligence, robotics and remote sensing.

About the Author-CHUN-HSIUNG FANG is a Ph.D. student with the Department of Electrical Engineering, National Taiwan University, Taiwan, ROC. His research interests are machine vision, pattern recognition and robotics. 